\title{
Prevalência de HIV, papilomavírus humano e sífilis na Penitenciária Feminina da Capital, São Paulo, 1997-1998
}

\author{
HIV, HPV, and syphilis prevalence in a women's \\ penitentiary in the city of São Paulo, 1997-1998
}

Fernanda Lopes 1

Maria do Rosário Dias de Oliveira Latorre 1

Antonio Carlos Campos Pignatari 2

Cassia Maria Buchalla 1

\footnotetext{
1 Departamento de Epidemiologia, Faculdade de Saúde Pública, Universidade de São Paulo. Av. Dr. Arnaldo 715, sala 40 (subsolo), São Paulo, SP 01246-904, Brasil.

2 Escola Paulista de Medicina, Universidade Federal de São Paulo. Rua Botucatu 715, 7 o andar, São Paulo, SP 04023-062, Brasil.
}

Abstract Incarcerated women as a group are particularly vulnerable to infections. The lack of public programs for prevention, early diagnosis, and treatment contribute to the increase in the incidence and prevalence of diseases in general and especially sexually transmitted diseases. This article aims to estimate the preval ence of infection by the human immunodeficiency virus (HIV), human papillomavirus (HPV), and syphil is among inmates at the Women's Penitentiary in the State capital of São Paulo, Brazil. All inmates were invited to partici pate in the study, which was divided into two stages: 1. STD/AIDS preventive workshops including interviews and 2. laboratory tests. The interview covered knowledge of STD/AIDS, risk behavior, and individual reproductive health history. A total of 262 women, with a mean age of 32.4 years and limited schooling, participated in more than one stage of the study. Prevalence rates were $14.5 \%$ for HIV, 16.3\% for high-oncogenic-risk HPV probes, 4.8\% for low-oncogenic-risk HPV probes, and 5.7\% for syphilis. The authors conclude that STD/HIV constitute a serious health problem in the prison system, requiring urgent preventive measures.

Key words HIV; Acquired Immunodeficiency Syndrome; Human Papillomavirus; Syphilis; Women's Health

Resumo Mulheres encarceradas constituem um grupo especialmente vulnerável a infecções. A inexistência de programas oficiais de diagnóstico precoce, tratamento e prevenção contribuem para o aumento da incidência e prevalência de doenças, sobretudo as transmiti das sexualmente. Este artigo obj etiva esti mar a prevalência de infecção por HIV, HPV e sífilis em mulheres da Penitenciária Feminina da Capital - São Paulo/Brasil. Todas as mulheres da unidade prisional foram convidadas a participar do estudo que foi dividido em duas fases: (1) oficinas de prevenção às DST/AIDS e entrevista; (2) exames laboratoriais. A entrevista abordou conhecimento sobre as DST/AIDS, comportamento de risco e história reprodutiva. O total de 262 mul heres, com idade média de 32,4 anos e baixo nível de escolaridade, participou em mais de uma etapa do estudo. Foram observadas prevalências de 14,5\% para infecção por HIV, 16,3\% com sondas de HPV dealto potencial oncogênico, 4,8\% com sondas de HPV de baixo potencial oncogêni co e 5,7\% para sífilis. Conclui-se que as DST/HIV constituem grave problema de saúde no sistema penitenciário quejustificam medidas preventivas urgentes.

Palavras-chave HIV; Síndrome de Imunodeficiência Adquirida; Papilomavírus Humano; Sífilis; Saúde da Mulher 


\section{Introdução}

Segundo a Organização Mundial de Saúde (OMS) a incidência global de DST (doenças sexualmente transmissíveis) curáveis é de, aproximadamente, 333 milhões/ano. Dois terços de todos os casos ocorrem em pessoas com idade inferior a 25 anos e, em algumas populações, a maioria dos indivíduos adultos está infectada por um ou mais patógenos. Desde o aparecimento da AIDS, tanto as DST clássicas, como a sífilis e gonorréia, como as (re)emergentes como a infecção por papilomavírus humano (HPV), passaram a ser melhor estudadas (Buchalla, 1995; Dallabetta et al., 1997; Naud, 1993).

Embora a infecção por HPV seja conhecida há séculos como precursora de lesões benignas denominadas verrugas genitais, existem evidências de que ela também esteja associada ao desenvolvimento do câncer de colo uterino. Atualmente, em populações sexual mente ativas, sua prevalência excede à de gonorréia e de clamídia (Adimora et al.,1994; Franco, 1991; Naud, 1993). No caso de mulheres HIV-positivas, alguns autores sugerem o aumento do risco tanto para a aquisição/ persistência da infecção, quanto para progressão de lesões associadas (Meuler et al., 1992; Sampaio Neto et al., 1997; Seek et al., 1994; Vernon et al., 1994).

$O$ crescimento da incidência de DST, sobretudo entre mulheres jovens, conferiu ao Brasil o mais rápido aumento de casos de AIDS na população feminina descrito no mundo (Panos Institute, 1993). Se, na década de 80, dentre os casos notificados, a razão de sexo era de 27 homens para cada caso em mulher, no final dos anos 90 esta relação passou a ser $3 / 1$. No Município de São Paulo, por exemplo, desde o início da década de 90, a AIDS tem sido uma das principais causas de morte em mulheres com idade entre 25 e 49 anos (MS, 1998; SES-SP, 1998).

A rápida disseminação de DST em grupos de menor poder aquisitivo é melhor caracterizada quando se avalia sua ocorrência em populações confinadas. Nos Estados Unidos, em 1995, entre as mulheres que ingressaram no sistema prisional, $27 \%$ estavam infectadas por clamídia e 8\% tinham gonorréia. Em 1996, 17\% dos casos de AIDS notificados neste país eram oriundos do sistema prisional. Em 1997, foi estimado que cerca de 8.900 presos tinham AIDS e que 35 a 47 mil eram portadores do HIV (Skolnick, 1998). Somado a isto, no período de 1996 a 1999, foi observada uma variação de 3 a 22\% na prevalência de sífilis entre mulheres ingressantes no sistema (Altman, 1999). No Brasil existem poucos dados sobre incidência, prevalência e outros aspectos epidemiológicos rela- cionados às DST-HIV/ AI DS na população prisional, em particular, no efetivo feminino.

A necessidade de conhecer a prevalência da infecção por HIV, sífilis e HPV entre as mulheres da Penitenciária Feminina da Capital (PFC), justifica-se tanto por ser esta uma população mais exposta aos riscos, como para estimular uma resposta do serviço de saúde e de prevenção prestado a essa população, especialmente por estar em adoção de regime de visita íntima.

\section{Material e métodos}

Estudo transversal realizado no período de agosto de 1997 a fevereiro de 1998, na PFC, unidade do Complexo Penitenciário do Carandiru, Município de São Paulo. Todas as 414 muIheres que estavam na unidade entre 10 de agosto e 8 de setembro de 1997 foram convidadas a participar das atividades do estudo. Desse total, por determinação da diretoria da unidade prisional, foram excluídas 45 mulheres: 12 por apresentarem problemas de saúde, por estarem grávidas e/ ou no puerpério, quatro por serem recém-egressas do hospital psiquiátrico e outras 29 que estavam na iminência de serem libertadas. Trezentos e sessenta e nove mulheres foram efetivamente convidadas e 316 participaram de, no mínimo, uma etapa. O estudo foi realizado em cinco etapas, a saber:

1) Sensibilização e intervenção educativa: por meio de oficinas semanais de trabalho em grupo, em que foram apresentados e discutidos temas como sexo seguro e prevenção à DST/ AIDS. Cada grupo participou de três oficinas totalizando nove horas de atividade por grupo;

2) Consulta individual sobre interesse na realização dos exames e oficialização do consentimento de participação. Foram dadas explicações sobre os objetivos do estudo, metodologia, riscos, inexistência de implicações em caso de participação ou recusa, garantia de confidencialidade de resultados e autonomia para desistir a qualquer momento, sem prejuízo pessoal. Também foi informado que as infectadas seriam encaminhadas para tratamento e que este seria conduzido sob a responsabilidade da unidade prisional. Aquelas que demonstraram interesse/ disposição tiveram o consentimento de participação oficializado;

3) Entrevistas individuais com duração média de trinta minutos, conduzidas de acordo com um formulário epidemiológico pré-testado, com questões fechadas;

4) Coleta de amostras clínicas para análise. Para a pesquisa de anticorpos anti-HIV uma amostra de $10 \mathrm{ml}$ de sangue foi submetida a 
duas reações de ELISA (Enzyme Linked Imunosorbent Assay) realizadas no Laboratório de Imunossorologia do Centro de Referência e Treinamento em DST/AIDS da Secretaria Estadual de Saúde de São Paulo (SES-SP). Os resultados positivos foram confirmados por Western Blot e/ ou Imunoflorescência Indireta. Para a pesquisa de anticorpos anti-treponêmicos e realização do VDRL (Venereal Disease Research Laboratories Tests), outra amostra de $10 \mathrm{ml}$ de sangue foi analisada no Laboratório de Sorologia da Associação Fundo de Incentivo à Psicofarmacologia da Universidade Federal de São Paulo (UNIFESP). A detecção do DNA/HPV foi feita por Hibridização Molecular por Captura Híbrida utilizando Digene HPV Test - Hybrid Capture. A análise do material coletado foi realizada no Laboratório de Microbiologia Molecular da Disciplina de Doenças Infecciosas e Parasitárias da UNIFESP;

5) Entrega individual de resultados e encaminhamentos. As mulheres HPV-positivas foram encaminhadas para exame colposcópico na Fundação Oncocentro de São Paulo. Aquelas que apresentaram sorologia positiva para HIV fizeram contagem de CD4/CD8 (exame realizado no Laboratório de Retrovirologia da Disciplina de Doenças Infecciosas e Parasitárias da UNIFESP). Os casos de sífilis ativa e os demais casos de DST curáveis foram encaminhados para o Serviço de Saúde da unidade prisional para tratamento e acompanhamento.

O protocolo de pesquisa foi aprovado pela Comissão de Ética em Pesquisa da Faculdade de Saúde Pública da Universidade de São Paulo (USP) e todas as etapas supracitadas foram realizadas com cada participante, na ausência das autoridades carcerárias.

Os dados obtidos foram codificados e analisados utilizando o programa Epi Info, versão 6.0 (Dean et al., 1994).

\section{Resultados e discussão}

O caráter voluntário do estudo e as constantes flutuações da população encarcerada explicam a diferença na freqüência de participação em cada uma das etapas.

Dentre as 369 mulheres convidadas, 53 (14,4\%) recusaram-se a participar do estudo, o que resultou em um total 316 pessoas estudadas. Os motivos alegados para as recusas foram, em sua totalidade, falta de interesse e problemas pessoais.

No entanto, a participação não foi igual em todas as etapas. Das 316 que concordaram em participar da primeira etapa, 49 não tiveram interesse em ser entrevistadas e/ ou realizar os exames, e cinco optaram por apenas conceder entrevista. Das 262 mulheres restantes, algumas recusaram um dos exames oferecidos: seis $(2,3 \%)$ recusaram o teste anti-HIV, alegando falta de interesse em descobrir seu estado sorológico naquele momento e lugar; $12(4,6 \%)$ recusaram o teste para sífilis por terem realizado esse exame anteriormente, ou simplesmente por falta de interesse. Em outros lugares do mundo foram relatadas proporções semelhantes de recusa para testagem (Behrendt et al., 1994; Smith et al., 1991).

Trinta e quatro mulheres $(13,0 \%)$ recusaram-se a ter material ginecológico colhido, alegando constrangimento e/ ou falta de necessidade por estarem, há muito tempo, sem parceiro sexual, e 19 (7,2\%) foram libertadas ou transferidas antes que este material fosse colhido e que a entrevista fosse realizada. Até que todas as entrevistas fossem realizadas, outras $22 \mathrm{mu}$ Iheres $(9,0 \%)$ deixaram a unidade prisional.

A freqüência de participação em cada uma das etapas do estudo está apresentada na Tabela 1 .

A idade média das mulheres entrevistadas foi de 32,4 anos. O tempo de reclusão médio estimado foi de 2,9 anos. As características sócio-demográficas da população da PFC (Tabela 2) assemel haram-se tanto àquelas apresentadas pelas mulheres da Casa de Detenção Feminina do Tatuapé, São Paulo (CFL, 1997) quanto às apresentadas por mulheres livres residentes na Região Metropolitana de São Paulo.

Similaridades entre este grupo e populações femininas diversas também foram observadas no que diz respeito aos agravos de saúde relacionados ao aparel ho gênito-urinário (Tanaka et al.,1995). Na PFC, entre aquelas que ad-

Tabela 1

$\mathrm{N}$ úmero de participantes entre as mulheres da Penitenciária Feminina da Capital nas etapas do estudo. São Paulo, 1998.

\begin{tabular}{lc}
\hline Etapa & Número \\
\hline Sensibilização & 316 \\
Esclarecimentos e oficilização do consentimento & 267 \\
Coleta de material para exames & \\
Sangue para teste anti-HIV & 256 \\
Sangue para teste de sífilis & 244 \\
Escovado cérvico-vaginal para captura de DNA/HPV & 209 \\
Entrevista & 226 \\
\hline
\end{tabular}


Tabela 2

Características sócio-demográficas e relacionadas à vida sexual das mulheres entrevistadas. Penitenciária Feminina da Capital, São Paulo, 1998.

\begin{tabular}{|c|c|c|}
\hline Variáveis/categoria & $n$ & $\%$ \\
\hline \multicolumn{3}{|l|}{ Idade } \\
\hline $20-31$ & 125 & 55,4 \\
\hline $32-43$ & 77 & 34,0 \\
\hline 44 ou mais & 24 & 10,6 \\
\hline \multicolumn{3}{|l|}{ Cor da pele } \\
\hline Negra & 132 & 58,4 \\
\hline Branca & 94 & 41,6 \\
\hline \multicolumn{3}{|l|}{ Nível de escolaridade } \\
\hline Nenhum & 26 & 11,5 \\
\hline Ensino Fundamental & 160 & 70,7 \\
\hline Ensino Médio & 30 & 13,3 \\
\hline Superior & 10 & 4,5 \\
\hline \multicolumn{3}{|c|}{ Idade de início da vida sexual (anos) } \\
\hline$\leq 16$ & 153 & 68,0 \\
\hline 17 ou mais & 72 & 32,0 \\
\hline \multicolumn{3}{|c|}{ Número de parceiros sexuais durante a vida } \\
\hline$\leq 3$ & 124 & 55,1 \\
\hline 4 ou mais & 101 & 44,9 \\
\hline \multicolumn{3}{|l|}{ História prévia de DST } \\
\hline Sim & 94 & 44,1 \\
\hline Não & 119 & 55,9 \\
\hline
\end{tabular}

mitiram história prévia de DST, metade identificou-a como candidíase, $17,0 \%$ sífilis, $16,0 \%$ verruga genital e 11,7\% gonorréia.

Um número elevado de mulheres fez uso de drogas, sendo comum a associação de mais de uma delas (Tabela 3). Estudos realizados em outras unidades prisionais do Estado de São Paulo também mostraram ser el evada a referência ao uso de drogas pelas mulheres e/ ou por seus parceiros. Em Santos, o uso de maconha foi referido por $36,4 \%$ das mulheres entrevistadas, o uso de cocaína inalada por $46,6 \%$, o uso de crack por $36,0 \%$ e de drogas injetáveis por 16,6\% (Tellini et al., 1998). Na Casa de Detenção Feminina do Tatuapé, a maconha foi citada como uma das drogas utilizadas por $44,0 \%$ das mulheres. Trinta e seis por cento citaram o crack e $20,0 \%$ as drogas injetáveis (CFL, 1997). O uso de drogas pelo(s) parceiro(s) também foi comumente citado. Entre as mulheres da PFC que nunca haviam usado drogas, 7,1\% relataram ter parceiro(s) usuário(s) de drogas injetáveis (UDI). Entre aquelas que usavam outras drogas que não as injetáveis, essa proporção foi de $10,8 \%$ e, entre as usuárias de drogas injetáveis, $51,6 \%$ relataram que seus parceiros também eram UDI (dados não apresentados).

As prevalências das infecções pesquisadas são apresentadas na Tabela 4, bem como o número de mulheres submeti das a cada exame.

A infecção por HIV foi observada em 14,5\% das mulheres testadas, foi maior que os $7,2 \%$ relatados por Carvalho et al. (1998), que realizaram testes em mulheres encarceradas na $\mathrm{Ci}$ dade do Rio de Janeiro, e menor que os $26,0 \%$ observados por Ferreira (1997) que estudou a população da mesma unidade prisional entre 1994 e 1995. Este valor, contudo, apresentou semel hança ao observado em outros estudos envolvendo mulheres encarceradas, tais como o de Glassbrenner (1986), realizado em Maryland, Estados Unidos, e o de Benetucci et al. (1988), realizado na Argentina, que estimaram a prevalência da infecção em 15 e 14,0\%, respectivamente. Também foi semelhante ao observado em vários estudos realizados junto a homens mantidos em regime de reclusão em diferentes unidades do Complexo Penitenciário do Carandiru, o mesmo que abriga a unidade feminina cujas mulheres participaram desta pesquisa. Coscina (1992) relatou $12,5 \%$ de positividade para tal infecção; Rozman (1995) relatou 16,0\%; Kallás (1996) 13,7\%; e Massad et al. (1999) relataram 16,0\%. Comparando este estudo ao de Ferreira (1997), pôde-se considerar, além da característica flutuante da população, a possibilidade de recusa entre as mulheres que já conheciam seu estado sorológico ou que apresentavam alta percepção sobre comportamentos de risco. Cabe ressaltar que, embora 19 das mulheres cujo resultado foi reagente na pesquisa de anticorpos anti-HIV já conhecessem seu estado sorológico antes de participarem deste estudo (51,3\%), somente duas (com AIDS) eram acompanhadas por médico infectologista.

Em relação à cor da pele das mulheres que apresentaram resultado positivo em qualquer um dos exames, a porcentagem de negras foi sempre maior que $76,0 \%$, com exceção das portadoras de DNA/HPV de alto risco. Tendo sido identificadas desigualdades na qualidade de vida dos diferentes grupos populacionais norte-americanos, desde 1993, a variável raça/ etnia integra as estatísticas nacionais de saúde funcionando, principalmente, como um marcador de risco social. De acordo com dados do Centers for Di sease Control and Prevention (CDC) citados por Mandell (1997), em 1995, $56,0 \%$ dos casos de AIDS em mulheres norteamericanas eram atribuídos a mulheres negras. 
Tanto no Brasil quanto em outras partes do mundo, ser negro é estar submetido a condições desfavoráveis de viver e morrer sem que isso seja levado em consideração e, para estes indivíduos, o fato de ser do sexo feminino é ainda mais complicado (Barbosa, 1998; Cunha, 1995; Maio \& Santos, 1996; Oliveira, 1995). Exemplificando, enquanto $32,0 \%$ dos homens HIV-positivos da Casa de Detenção do Estado de São Paulo em 1996 eram negros (Kallás, 1996), entre as soropositivas da PFC, $77 \%$ eram mulheres negras. Assim, além de as prisões concentrarem indivíduos negros e pobres que não puderam atingir os patamares mínimos para o acesso a bens culturais e/ ou de serviços, concede-Ihes o direito à participação no grupo dos "especialmente vulneráveis" às doenças infecto-contagiosas, como é o caso do HIV/AIDS.

A prevalência de sífilis ativa na população estudada (5,7\%) foi inferior à observada por Miranda et al. (1998) na Penitenciária Feminina de Vitória, Espírito Santo (15,7\%). Porém, os dados obtidos na PFC não se distanciam daqueles gerados por um rastreamento para DST no efetivo feminino de uma das maiores cadeias da Região Metropolitana de Nova Orleans, Estados Unidos, em 1992, onde foi determinada uma prevalência de 3,1\% de sífilis (Beltrami, 1997).

A presença de DNA/HPV, sem distinção em grupos de al to ou baixo potencial oncogênico, foi identificada em 19,1\% das amostras testadas. Esses resultados podem ser comparados aos 19,5\% observados por Czeglédy et al. (1992) e aos $24,5 \%$ observados por Seck et al . (1994), em mulheres livres usuárias de clínicas de DST no Quênia e no Senegal, respectivamente. Dado que a prevalência de DST nos países da África é muito alta, esta semelhança entre taxas de infecção em mulheres livres, sexualmente ativas e mulheres reclusas e sem atividade sexual confirma a importância do diagnóstico precoce e tratamento da infecção.

Ainda que os achados relacionados ao perfil das mulheres infectadas por HPV se contraponham aos dados da literatura que apontam para a alta prevalência de lesões verrucóides em mulheres jovens e sexualmente ativas, eles apresentam semelhança com aqueles observados por Gonçalves (1998), que estudou mulheres HIV-positivas da Cidade de Santos, São Paulo. Segundo a autora, as mulheres soropositivas mais velhas mostraram-se mais infectadas por HPV de baixo risco.

Estudos realizados em grupos de mulheres HIV-positivas mostram a alta prevalência de infecções virais e fúngicas em seu trato genital, com destaque para a infeção por HPV e Candida sp. A maioria dos estudos sobre a prevalên-
Tabela 3

Distribuição do tipo de droga utilizado pelas mulheres entrevistadas e por seu(s) parceiro(s). Penitenciária Feminina da Capital, São Paulo, 1998.

\begin{tabular}{lrc}
\hline Variáveis/categoria & $n$ & $\%$ \\
\hline Tipo de droga ou via de uso & & \\
$\quad$ Cigarro & 170 & 86,0 \\
Maconha & 120 & 60,1 \\
Inalatórias & 89 & 45,0 \\
Crack & 71 & 36,0 \\
Álcool & 57 & 28,8 \\
Injetáveis & 31 & 15,6 \\
Seringa compartilhada & & \\
Sim & 16 & 55,2 \\
Não & 13 & 44,8 \\
Uso de drogas injetáveis pelo(s) parceiro(s) & & \\
Sim & & \\
Não & 36 & 21,3 \\
\end{tabular}

Tabela 4

Prevalência de DST nas mulheres. Penitenciária Feminina da Capital, São Paulo, 1998.

\begin{tabular}{lcc}
\hline Variáveis/categoria & $n$ & $\%$ \\
\hline $\begin{array}{l}\text { Elisa para AC anti-HIV }(\mathrm{n}=256) \\
\quad \text { Reagente }\end{array}$ & 37 & 14,5 \\
VDRL + Elisa para Ac anti-treponêmicos $1(\mathrm{n}=244)$ & 14 & 5,7 \\
$\quad$ Reagente & 34 & 16,3 \\
$\begin{array}{l}\text { Captura Híbrida para HPV de alto risco }(\mathrm{n}=209) \\
\quad \text { Positivo }\end{array}$ & 10 & 4,8 \\
$\begin{array}{l}\text { Captura Híbrida para HPV de baixo risco }(\mathrm{n}=209) \\
\text { Positivo }\end{array}$ & & \\
\hline
\end{tabular}

10 diagnóstico de sífilis foi atribuído às pacientes com soro reagente ao VDRL e Elisa para anticorpos anti-treponêmicos.

cia de lesões vulvo-vaginais associadas à infecção por HPV em mulheres HIV-positivas, sugere um aumento da prevalência tanto das condilomatoses quanto das neoplasias intraepiteliais vulvar e vaginal neste grupo (Adimora et al., 1994; Gonçalves, 1998; Kreiss et al., 1992; Laga et al., 1992; Sampaio Neto et al., 1997; Spitzer et al., 1993; Vernon et al., 1994; Zur-Hausen, 1981). Em estudo realizado em Nova Iorque, Estados Unidos, 70,0\% das mulheres com AIDS 
tiveram infecção persistente por HPV. Entre as HIV-positivas assintomáticas, a prevalência de infecção por HPV foi de $22,0 \%$, não existindo trabalhos de segmento com mulheres encarceradas (Vermund et al., 1991).

Quanto ao binômio HIV/sífilis, sabe-se que o Treponema pallidum pode funcionar como um marcador da transmissão do HIV por via sexual, uma vez que, na presença de lesões ulcerativas (sífilis recente, VDRL positivo com titulação maior ou igual a 1:16) a susceptibilidade às outras DST aumenta (Adimora et al., 1994; Dallabetta, 1997; Mandell, 1997; Naud, 1993). Todavia, a maior parte dos trabalhos sobre a interação HIV/T. pallidum deriva de ensaios clínicos em que a sífilis é uma das infecções oportunistas do quadro de AIDS (Mandell, 1997). Nos Estados Unidos, por exemplo, em 1992, foram registrados 33.973 casos de sífilis primária em mulheres HIV-positivas (CDC, 1993). Na PFC, entre as mulheres co-infectadas por HIV e sífilis, 33,3\% tiveram diagnóstico de sífilis recente. Os $66,7 \%$ restantes das mulheres tiveram diagnóstico laboratorial que comprova contato prévio com o T. pallidum, não podendo ser descartada a possibilidade de já apresentarem sinais de sífilis tardia com comprometimento cárdio-vascular e/ ou neurológico.

\section{Conclusões}

Com base nos resultados obtidos, pôde-se concluir que: (a) a alta prevalência de DST-HIV/ AIDS justifica a necessidade do diagnóstico precoce, da avaliação médica individual e da elaboração de um programa de educação/ prevenção; (b) a alta prevalência de infecção por HPV justifica a necessidade de uma atenção médica ginecológica mais eficaz na unidade prisional; (c) as baixas condições sócio-econômicas foram importantes marcadores de risco para as DST-HIV/ AIDS, refletindo o quadro de desigualdades estabelecido fora das prisões e confirmando a necessidade da implementação de programas de atenção primária à saúde que sejam adequados às diferentes realidades; (d) as características das mulheres da PFC, seu perfil de saúde e suas necessidades, são semelhantes às das mulheres encarceradas em outras partes do mundo, sendo viável, portanto, a adoção de estratégias de saúde anteriormente testadas. Para tanto, sugere-se que as autoridades penitenciárias devam se empenhar em oferecer testes sorológicos para as mulheres ingressantes, de forma a contribuir para a melhora de qualidade de vida no ambiente prisional, para a segurança/ preservação daqueles com quem, mais tarde, estas mulheres irão se relacionar e para diminuição dos custos diretos e indiretos com manutenção no período de permanência na prisão.

\section{Agradecimentos}

Agradecemos ao Conselho Nacional de Desenvolvimento Científico e Tecnológico e ao Departamento de Saúde do Sistema Penitenciário de São Paulo que financiaram a pesquisa; aos técnicos dos Laboratórios de Imunossorologia do Centro de Referência e Treinamento em DST-AIDS/ São Paulo e de Sorologia da Associação Fundo de Incentivo à Psicofarmacologia da Escola Paulista de Medicina da Universidade Federal de São Paulo; à equipe do Serviço de Citologia e Colposcopia da Fundação Oncocentro de São Paulo; e às psicólogas do Núcleo de Estudos em Prevenção de AIDS da Universidade de São Paulo. 
ADIMORA, A. A.; HAMILTON, H.; HOLMES, K. K. \& SPARTING, P. F., 1994. Sexually Transmitted Diseases. Singapore: Companion Handbook/International Editions.

ALTMAN, L. K., 1999. Much more AIDS in prisons than in general population. New York Times, New York, 9 jan. p.14.

BARBOSA, M. I. S., 1998. Racismo e Saúde. Tese de Doutorado, São Paulo: Faculdade de Saúde Pública, Universidade de São Paulo.

BEHRENDT, C.; KENDING, N.; DAMBITA, C.; HORMAN, J .; LAWLOR, J. \& VLAHOV, D., 1994. Voluntary testing for Human Immunodeficiency Virus (HIV) in a prison population with high prevalence of HIV. American Journal of Epidemiology, 139:918-926.

BELTRAMI, J. F.; COHEN, D. A. A.; HAMRICK, J. T. \& FARLEY, T. A. A., 1997. Rapid screening and treatment for sexually transmitted diseases in arrestees: A feasible control measure. American Journal of Public Health, 87:1423-1426.

BENETUCCI, J.; ASTARLOA, L.; MULTARE, S.; ZARATE, S.; MASSIO, E. \& RUBENS, J., 1988. Prevalence of infection among a close population of prisoners in Argentina. In: IV International Conference on AIDS, Abstracts Book, p. 313. Stockholm: Swedish Ministry of Health and Social Affairs/ National Bacteriological Laboratory/ Karoliska Institute/ World Health Organization.

BUCHALLA, C. M., 1995. AIDS: O surgimento e a evolução da doença. In: Velhos e Novos Males da Saúdeno Brasil: A Evolução do País e de suas Doenças (C. A. Monteiro, org.), pp. 331-345, São Paulo: Editora Hucitec/Núcleo de Pesquisas Epidemiológicas em Nutrição e Saúde, Universidade de São Paulo.

CARVALHO, M. L.; BIONDI, E. J. \&VEIGA, L. P., 1998. Estudo transversal sobre prevalência da infecção pelo HIV no sistema penitenciário do Rio de Janeiro, 1997. In: IV Congresso Brasileiro de Epidemiologia, Resumos, p. 194. Rio de Janeiro: ABRASCO.

CDC (Centers for Disease Control and Prevention), 1993. Use of race and ethnicity in public health surveillance. MMWR, 42. 12 November 1998 বttp:// www.cdc.gov/ epo/mmwr/preview/mmw rhtml/00021729.htm>.

CFL (Coletivo de Feministas Lésbicas), 1997. Prevenção do HIV/AIDS na Casa de Detenção Feminina do Tatuapé - São Paulo. Relatório de Pesquisa. São Paulo: Programa de DST/AIDS, Secretaria de Estado da Saúde de São Paulo/ Coordenação Nacional de DST/AIDS, Ministério da Saúde.

COSCINA, A. L., 1992. Pesquisa de Anticorpos para HIV-1 e HIV-2 em Presidiários Brasileiros. Dissertação de Mestrado, São Paulo: Universidade Federal de São Paulo, Escola Paulista de Medicina.

CUNHA, E. M. G. P., 1995. Raça: Um aspecto de iniqüidade esquecido na saúde do Brasil? In: III Congresso Brasileiro de Epidemiologia, Resumos, p. 442, Salvador: ABRASCO.

CZEGLÉDY, J.; ROGO, K. O.; EVANDER, M. \&WADELL, G., 1992. High-risk human papillomavirus types in cytologically normal cervical scrapes from Kenya. Medical Microbiology and Immunology, 180:321-326.
DALLABETTA, G.; LAGA, M. \& LAMPTEY, P., 1997. Controle de Doenças Sexualmente Transmissíveis: Manual dePlanejamento e Coordenação de Programas. Rio de Janeiro: Te Corá Editora/ Associação Saúde da Família.

DEAN, A. G.; DEAN, J. A.; COULOMBIER, D.; BRENDEL, K. A.; SMITH, D. C.; BURTON, A. H.; DICKER, R. C.; SULLIVAN, K.; FAGAN, R. F. \& ARNER, T. G., 1994. Epi Info, Version 6: A Word Processing, Database, and Statistics Program for Epidemiology on Microcomputers. Atlanta: Centers for Disease Control and Prevention.

FERREIRA, M. M. C., 1997. Infecção pel os Retrovírus HIV-1, HTLV-1 e HTLV-II na População Feminina da Penitenciária do Estado de São Paulo. Prevalência, Fatores de Risco e Conhecimento desse Risco. Tese de Doutorado, São Paulo: Faculdade de Saúde Pública, Universidade de São Paulo.

FRANCO, E. L., 1991. Viral etiology of cervical cancer: A critique of the evidence. Journal of Infectious Diseases, 13:1195-206.

GLASSBRENNER, J., 1986. Prisons confront dilemma of inmates with AIDS. JAMA, 255:2399-2404.

GONÇALVES, M. A. G., 1998. Estudo da Prevalência de Papiloma Vírus Humano (HPV) e de Neoplasia Intraepitelial Genital em Amostra de Mulheres HIV Positivas da Cidade de Santos - São Paulo, Brasil. Tese de Doutorado, São Paulo: Faculdade de Saúde Pública, Universidade de São Paulo.

KALLÁS, E. G., 1996. Prevalência eldentificação dos Fatores de Risco para Infecção pel o Vírus da Imunodeficiência Humana na Casa de Detenção de São Paulo. Dissertação de Mestrado, São Paulo: Escola Paulista de Medicina, Universidade Federal de São Paulo.

KREISS, J. K.; KIVIAT, N. B.; PLUMMER, F. A.; ROBERTS, P. L.; WAIYAKI, P. \& NGUGI, E., 1992. Human immmunodeficiency virus, human papillomavirus, and cervical intraephitelial neoplasia in Nairobi Prostitutes. Sexually Transmitted Diseases, 19:54-59.

LAGA, M.; ICENOGLE, J. P.; MARSELLA, R.; MANOKA, A. T.; NZILA, N. \& RYDER, R. W., 1992. Genital papillomavirus infection and cervical dysplasia Opportunistic complications of HIV infection. International Journal of Cancer, 50:45-48.

MAIO, M. C. \& SANTOS, R. V., 1996. Raça, Ciência e Sociedade. Rio de Janeiro: Fundação Oswaldo Cruz/Centro Cultural Banco do Brasil.

MANDELL, G. L., 1997. AIDS. Atlas of Infectious Diseases, v. 1. Philadel phia: Current Medicine.

MASSAD, E.; ROZM AN, M .; AZEVEDO, R. S.; SILVEIRA, A. S.; TAKEY, K.; YAM AMOTO, Y. I.; STRAZZA, L.; FERREIRA, M. M. \& BURATTINI, M. N., 1999. Seroprevalence of HIV, HCV and syphilis in brazilian prisoners: Preponderance of parenteral transmission. European Journal of Epidemiology, 15: 439-445.

MEULEN, J. T.; EBERHARDT, H. C.; LUANDE, J.; MGAYA, H. N.; CHANG-CLAUDE, J. \& MITIRO, H., 1992. Human Papillomavirus (HPV) infection, HIV infection and cervical cancer in Tanzania, East Africa. International Journal of Cancer, 51: 515-521. 
MIRANDA, A. E.;VARGAS, P. M. \& VIANA, M. C., 1998. Prevalência de infecção por HIV, HTLV I, HBV, HCV e sífilis na Penitenciária Feminina do Espírito Santo. Jornal Brasile ro de Doenças SexualmenteTransmissíveis, 10:63.

MS (Ministério da Saúde), 1998. Aids no Brasil: Um Esforço Conjunto, Governo e Soci edade. Brasília: Coordenação Nacional de DST/AIDS, Ministério da Saúde.

NAUD, P., 1993. DST \& AIDS. Porto Alegre: Artes Médicas.

OLIVEIRA, F., 1995. Sexo/gênero e raça/ etnia em epidemiologia: Uma articulação necessária em busca da eqüidade. In: III Congresso Brasileiro de Epidemiologia, Resumos, p. 442. Salvador: ABRASCO.

PANOS INSTITUTE, 1993. Dossiê Panos - A Tripla Ameaça: Mulheres eAIDS. Rio de Janeiro: Associação Brasileira de Interdisciplinar de AIDS/ Recife: SOS Corpo.

ROZM AN, M. A., 1995. AIDS e Tuberculose na Casa de Detenção de São Paulo. Dissertação de Mestrado, São Paulo: Faculdade de Medicina, Universidade de São Paulo.

SAM PAIO NETO, L. F.; CANÇADO, R. R.; FARIA, M., FERREIRA, R. M. V.; DIAS J r., A. \& REIS, E., 1997. Estudo da prevalência de doenças sexualmente transmissíveis em grupos de alto risco. Revista Brasileira de Doenças Sexualmente Transmissíveis, 2:23-32.

SECK, A. C.; FAYE, M. A.; CRITCHLOW, C. W.; MBAYE, A. D.; KUYPERS, J.; WOTO-GAYE, G.; LANGLEY, C.; DE, E. B.; HOLMES, K. K. \& KIVIAT, N. B., 1994. Cervical intraepithelial neoplasia and human papillomavirus infection among Senegalese women seropositive for HIV-1 or HIV-2 or seronegative for HIV. International Journal of STD \& AIDS, 5:189-193.

SES-SP (Secretaria de Estado da Saúde de São Paulo), 1998. Boletim Epidemiológico AIDS, junho 1998. São Paulo: Centro de Vigilância Epidemiológica, SES-SP.
SKOLNICK, A. A., 1998. Look behind bars for key to control of STDs. JAMA, 279:97-99.

SMITH, P. F.; MIKL, J.; TRUMAN, B. I.; LESSNER, L.; LEHMAN, J. S. \& STEVENS, R. W., 1991. HIV infection among women entering the New York State Correctional System. American Journal of Public Health, 81(Sup.):35-40.

SPITZER, M.; BRENNESSEL, D.; SELTZER, V. L.; SILVER, L. \& LOX, M. S., 1993. Is human papillomavirus-releated disease an independent risk factor for human immunodeficiency virus infection? Gynecologic Oncology, 49:243-246.

TANAKA, A. C. D. A.; SCHOR, N.; ALVARENGA, A. T. \& SIQUEIRA, A. F., 1995. Morbidade feminina de residentes na região sul do Município de São Paulo. In: III Congresso Brasileiro de Epidemiologia, Resumos, p. 357. Salvador: ABRASCO.

TELLINI, R. M. C.; CARVALHO, E. L.; GOMES, E.; EBNER, F. V.; CASTRO, M. T. F. \& ELLO, L. B., 1998 Estudo sobre a Prevalência de HIV e Outras Doenças Sexualmente Transmissíveis entre Presidiárias de Santos, São Paulo. Relatório de Pesquisa. São Paulo: AIDS Control and Prevention Project/Family Health International.

VERMUND, S. H.; KELLEY, K. F.; KLEIN, R. S.; FEINGOLD, A. R.; SCHREIBER, K. \& MUNK, G., 1991. High risk of human papillomavirus infection and cervical squamous intraepithelial lesions among women with symptomatic human immunodeficiency virus infection. American Journal of Obstetric and Gynecology, 165:392-400.

VERNON, S. D.; REEVES, W. C.; CLANCY, K. A.; LAGA, M.; ST. LOUIS, M.; GARY J r., H. E.; RYDER, R. W.; MANOKA, A. T. \& ICENOGLE, J. P., 1994. A longitudinal study of human papillomavirus DNA detection in human immunodeficiency virus type 1-seropositive and -seronegative women. Journal of Infectious Diseases, 169: 1108-1112.

ZUR-HAUSEN, H.; DE VILLIERS, E. M. \& GISSMAN, L., 1981. Papillomavirus infections and human genital cancer. Gynecological Oncology, 12(Sup.): S124-S128. 